\title{
Atividade inseticida e repelente de extrato e pó de sementes de Anethum graveolens e Azadirachta indica frente ao Sitophilus zeamais
}

\author{
Insecticidal activity and repellent of extract and powder of Anethum graveolens and Azadirachta \\ indica seeds against Sitophilus zeamais
}

B. Albiero; G. Freiberger; A. Biasi Vanin*

Ciências Exatas e da Terra, Universidade do Oeste de Santa Catarina, 89600-000, Joaçaba-SC, Brasil

*adriana.vanin@unoesc.edu.br

(Recebido em 31 de janeiro de 2020; aceito em 14 de abril de 2020)

\begin{abstract}
Sitophilus zeamais conhecido como gorgulho do milho é uma praga que afeta a qualidade de grãos armazenados. $\mathrm{O}$ uso de inseticidas químico é o método mais utilizado para o controle desta praga causando impactos ao meio ambiente. Neste sentido, meios menos agressivos são avaliados para o controle de insetos por meio de plantas com compostos de interesse, como extratos, que possuem metabólitos secundários que atuam na defesa das plantas. Portanto, o objetivo foi avaliar o potencial inseticida e repelente de plantas presentes na região Sul. A mortalidade e repelência de adultos $S$. zeamais foi avaliada em placas de Petri contendo grãos de milho tratados com extrato e pó de Anethum graveolens L. (endro) e Azadirachta indica A. Juss. (nim) em condições de laboratório. Todos os tratamentos afetaram a sobrevivência dos insetos. O melhor resultado foi para o extrato de A. graveolens, que apresentou $\mathrm{DL}_{50}=139,16 \mu \mathrm{L} /$ placa, menor valor dentre os tratamentos. A dose de $0,25 \mathrm{~g}$ de pó de endro/ $10 \mathrm{~g}$ de milho causo $100 \%$ dos insetos dos insetos, conferindo potencial inseticida. Quanto a repelência, o extrato de A. graveolens atingiu o maior Índice de Preferência (I.P.) repelindo $100 \%$ dos insetos na dose de $1,35 \mu \mathrm{L} / \mathrm{cm}^{2}$. O pó de A. graveolens repeliu a partir de $0,15 \mathrm{~g}$ e o extrato de $A$. indica foi repelente nas doses de $2,29 \mu \mathrm{L} / \mathrm{cm}^{2}$ e $3,95 \mu \mathrm{L} / \mathrm{cm}^{2}$, e atraente nas menores, $0,64 \mu \mathrm{L} / \mathrm{cm}^{2}$ e $1,37 \mu \mathrm{L} / \mathrm{cm}^{2}$. A utilização de extratos e pó de endro e nim constituem uma alternativa de controle sobre o gorgulho do milho.

Palavras-chave: Sitophilus zeamais, inseticida, repelente.
\end{abstract}

Sitophilus zeamais known as maize weevil is a pest that affects the quality of stored grains. A common method used for control is chemical insecticides that affect the environment. In this regard, less aggressive ways of controlling insects through plants containing compounds of interest, such as extracts, which have secondary metabolites that act in the defense of plants, are being researched. Therefore, the objective was to evaluate the insecticide and repellent potential of plants present in the South region. The repellency and survival of $S$. zeamais were evaluated under laboratory conditions with maize grains treated with powder obtained of Anethum graveolens L. (dill) and extract of A. graveolens and Azadirachta indica A. Juss. (neem). All treatment possessed insecticidal effect of $S$. zeamais. The extract that most affected the survival of the pest was A. graveolens, with $\mathrm{LD}_{50}$ of $139.16 \mu \mathrm{L}$. Obtained $0.25 \mathrm{~g}$ of dill powder/10 $\mathrm{g}$ of maize to affect the survival of $100 \%$ of insects, conferring insecticide potential. Regarding repellency, A. graveolens extract reached the highest Behavior Index (B.I.) and repelled $100 \%$ of insects with $1.35 \mu \mathrm{L} / \mathrm{cm}^{2}$. A. graveolens powder repelled from $0.15 \mathrm{~g}$ and A. indica extract was repellent at doses of $2.29 \mu \mathrm{L} / \mathrm{cm}^{2}$ and $3.95 \mu \mathrm{L} / \mathrm{cm}^{2}$, and attractive at the smallest, $0.64 \mu \mathrm{L} / \mathrm{cm}^{2}$ and $1,37 \mu \mathrm{L} / \mathrm{cm}^{2}$. The use of dill extracts and dill powder is an alternative to control over $S$. zeamais.

Keywords: Sitophilus zeamais, insecticide, repellent.

\section{INTRODUÇÃO}

O milho (Zea mays L.) é um dos mais antigos grãos cultivados e uma das mais importantes culturas produzidas no Brasil e no mundo. Em razão da expansão geográfica na região Centro-oeste do país e a adoção e difusão das inovações tecnológicas, atualmente o Brasil é um dos maiores produtores e exportadores de produtos agropecuários do mundo. Os grãos, principalmente a soja e o milho, testemunharam o crescimento rápido da produção e da produtividade [1]. Por apresentar alto valor nutricional, está presente intensamente na alimentação humana e animal, além de ser 
amplamente utilizado na indústria pelo seu baixo custo e viabilidade de cultivo [2]. A preservação da qualidade dos grãos é um grande desafio, principalmente durante a armazenagem, em que cerca de $10 \%$ da produção é atacada por insetos-praga, especialmente carunchos e gorgulhos [2]. As pragas causam uma degradação irreversível na massa dos grãos, reduzindo assim seu peso, qualidade, vigor de germinação, percentual de gordura do cereal bem como seu valor comercial, além de depositarem grandes quantidades de resíduos no estoque [4].

Sitophilus zeamais Motschulsky, 1885 (Coleoptera: Curculionidae) é um dos insetos mais prejudiciais aos grãos de milho, arroz e trigo [5]. Esse inseto possui elevado potencial reprodutivo, gera muitos descendentes a curto prazo, o que dificulta o seu controle e, é classificado como praga primária interna pois Sitophilus zeamais perfura facilmente o grão e coloca os ovos no interior, deixando orifícios em suas camadas que se tornam suscetíveis a outros agentes indesejáveis. Por ser uma espécie de infestação cruzada o gorgulho pode atacar o grão tanto na lavoura quanto no armazenamento e quando se encontram em elevada população migram para a lavoura, potencializando as perdas $[3,23]$.

O método mais comum para o controle de pragas é através de inseticidas ou repelentes químicos por serem de fácil manejo, baixo custo e alta eficácia. Os principais ingredientes ativos pertencem aos organofosforados, piretróides e precursores de fosfina. Porém, o uso intensivo de inseticidas químicos afeta negativamente o meio ambiente, gerando acúmulo de resíduos nos alimentos e resistência entre as espécies $[4,6]$. O controle de resíduos e o período de carência do produto devem ser observados a fim de amenizar os efeitos indesejados dos pesticidas. Os impasses decorrentes da utilização de inseticidas químicos apontam para a necessidade de buscar alternativas de manejo mais seletivos, eficientes e menos agressivos ao ecossistema [4, 6].

Uma alternativa para o controle químico são inseticidas naturais desenvolvidos a partir de plantas, cuja composição exerce atividade inseticida ou repelente, as quais podem ser utilizadas na forma de extrato, óleo essencial ou pó [6, 40]. Essas substâncias apresentam, geralmente, ausência de toxicidade a mamíferos, eficácia em baixas concentrações e, por serem obtidas de fontes renováveis, são rapidamente degradadas e não deixam resíduos [7, 40].

Os componentes orgânicos produzidos pelas plantas são classificados em dois grupos principais. Os metabólitos primários são responsáveis pela estruturação e armazenamento de energia, enquanto que os metabólitos secundários atuam na atração ou defesa contra insetos e patógenos através da sintetização de compostos como terpenos, terpenoides, fenilpropanoides, flavonoides e tiofenos [8].

A formação de metabólitos secundários ocorre por meio de metabólitos intermediários derivados da glicose, como o ácido chiquímico, precursor de fenilpropanoides e o ácido mevalônico, precursor de terpenoides [9].

Os terpenoides são classificados pelo número de carbonos, resultantes do número de moléculas de isopreno (2-metil-1,3-butadieno), como os monoterpenos (C10) e sesquiterpenos (C15) que são as substâncias mais frequentes em óleos essenciais e possuem importantes propriedades biológicas [11]. Os óleos essenciais são compostos voláteis extraídos das plantas e a maioria de seus componentes são derivados de fenilpropanoides e de terpenoides [10].

Dentre os terpenoides que apresentam atividade inseticida, os maiores representantes são os tetra-nor-triterpenos, conhecidos como limonóides os quais são comumente extraídos de plantas da família Meliaceae. Uma das espécies promissoras da família Meliaceae é a Azadirachta indica A. Juss., conhecida como nim, que apresenta baixa toxicidade e ampla distribuição na natureza [12]. A planta possui mais de 50 compostos terpenoides, sendo os principais a azadiractina, salanina, meliantrol e nimbolina, que podem estar presentes nas folhas, sementes e, principalmente frutos $[13,14]$.

Do mesmo modo, plantas aromáticas da família Apiaceae liberam grande quantidade de compostos voláteis que atuam como repelentes ou atrativos de inimigos naturais de insetos-praga, por meio de interações tritróficas [15]. Anethum graveolens L., conhecida por endro, é utilizada industrialmente por suas propriedades farmacológica, antifúngica e antimicrobiana. A semente desta espécie é a parte mais valorizada e seus principais componentes são o dilapiol, pertencente ao grupo dos fenilpropanoides; a carvona, um monoterpenoide; e o limoneno, um monoterpeno [16, 17]. 
Devido a importância do controle de insetos-praga nas lavouras e dos problemas apresentados pelos pesticidas sintéticos como persistência ambiental e efeitos tóxicos em organismos não alvo, é necessário o desenvolvimento de produtos menos agressivos e de mesma eficácia [24, 40].

Diante das propriedades biológicas de compostos produzidos por plantas, a pesquisa objetiva avaliar a atividade inseticida e repelente do extrato de $A$. indica e do extrato e pó das sementes de A. graveolens sob insetos $S$. zeamais em grãos armazenados.

\section{MATERIAL E MÉTODOS}

A metodologia baseou-se em Procópio et al. (2003) [18] com algumas adaptações. Para a realização dos ensaios foram utilizados extratos puros e pós das espécies a serem avaliadas. Os extratos foram obtidos por meio de destilação em Soxhlet, utilizando o éter de petróleo como solvente orgânico. O pó de A. graveolens foi obtido através da moagem das sementes da planta.

Para o processo de ovulação, 20 insetos adultos da espécie $S$. zeamais foram incubados em recipiente plástico e mantidos a $25^{\circ} \mathrm{C}$ durante 15 dias utilizando milho como substrato alimentar. Após esse período, os insetos adultos foram retirados do recipiente permanecendo somente os ovos que foram mantidos até a eclosão. Os insetos usados nos bioensaios tinham uma faixa etária de até 15 dias de vida.

\subsection{Avaliação do potencial inseticida}

No tratamento com extratos, foram utilizadas placas de Petri forradas com papel filtro impregnado com a dose do extrato a ser testada. Para os dois extratos foram realizados ensaios com doses crescentes em intervalos de 70 a $250 \mu \mathrm{L}$ para o extrato de endro e 170 a $800 \mu \mathrm{L}$ para o extrato de nim. Todos os ensaios foram realizados em triplicata, e uma placa sem extrato como testemunha. Em cada placa foram colocados 20 insetos e após 24 horas de inoculação contou-se os insetos mortos em cada placa.

Para avaliar o pó de endro, foram utilizadas placas de Petri contendo as quantidades de 0,15 , $0,075,0,0375,0,01875 \mathrm{~g}$ de pó vegetal/placa e uma placa sem pó, como testemunha. Em cada teste foram adicionados $10 \mathrm{~g}$ de substrato alimentar e 20 insetos, e após 5 dias foram contados os insetos mortos em cada placa.

Para a análise da eficácia dos extratos e do pó sobre os insetos utilizou-se a fórmula de Abbot em que Prates et al. (2019) [7]: Eficácia (\%) = mortalidade tratamento - mortalidade testemunha.

Foi aplicada regressão linear aos dados obtidos pela relação entre a mortalidade dos insetos e a dose da espécie testada. As doses letais (DL) foram determinadas através da análise Probit.

\subsection{Avaliação do potencial repelente}

A avaliação da repelência dos extratos e do pó foi realizada em triplicada, os insetos utilizados no teste ficaram por 3 horas sem contato com substrato alimentar antes dos ensaios. Foram montadas arenas, cada uma com uma placa de Petri ao centro e outras 4 ao redor. Foram colocados 30 insetos, não sexados, na placa central e $10 \mathrm{~g}$ de milho nas demais placas. Em duas placas de lados opostos foram acrescentadas doses letais de extratos ou de pó (tratamento), e as outras duas placas permaneceram apenas com substrato alimentar (testemunhas). Após 24 horas foi realizada a contagem dos insetos em cada placa.

A comparação dos tratamentos foi realizada através do Índice de Preferência (I.P.) em que [18]:

$$
\text { I.P. }=\frac{(\% \text { de insetos no tratamento })-(\% \text { de insetos na testemunha })}{(\% \text { de insetos no tratamento })+(\% \text { de insetos na testemunha })}
$$

I.P.: $-1,00$ a $-0,10=$ Teste repelente;

I.P.: $-0,10 \mathrm{a}+0,10=$ Teste neutro;

I.P.: $+0,10$ a $+1,00=$ Teste atraente. 


\section{RESULTADOS E DISCUSSÃO}

\subsection{Avaliação do potencial inseticida}

Os resultados para o extrato de sementes $A$. graveolens conferiram potencial inseticida à planta. A correlação entre as diferentes doses testadas e o percentual médio de mortalidade observado após 24 horas de exposição ao extrato pode ser observada na Figura 1, onde verifica-se um comportamento linear crescente $\left(\mathrm{R}^{2}=0,94\right)$, indicando um aumento da porcentagem de mortalidade dos insetos à medida em que se aumenta a dose de extrato. A DL $\mathrm{DL}_{50}$ calculada para o extrato de $A$. graveolens foi de $121,5 \pm 7,07 \mu \mathrm{L} /$ placa.

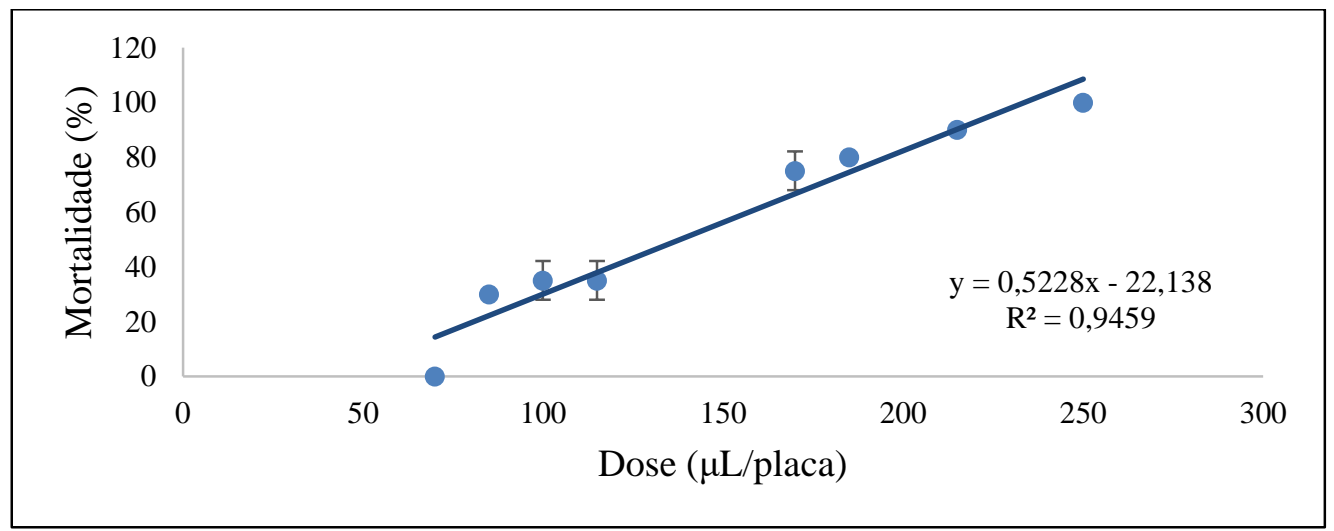

Figura 1: Mortalidade de Sitophilus zeamais tratado com extrato de Anethum graveolens.

Doses de extrato de $A$. graveolens próximas a $250 \mu \mathrm{L} /$ placa foram suficientes para se obter uma alta eficiência no controle de $S$. zeamais em condições experimentais. Tais doses possuem valores considerados elevados quando comparados com óleos essenciais obtidos de plantas que se desenvolvem na mesma região como o óleo de Cinamodendron dinisii que obteve $100 \%$ de mortalidade obtida com $115 \mu \mathrm{L} /$ placa [25] e baixo quando comparado com OE de folhas e ramos de Baccharis dracunculifolia D.C. (450 $\mu \mathrm{L} /$ placa) [26].

A ação inseticida pode ser explicada devido à composição química da planta. Dentre os principais compostos químicos de A. graveolens estão o dilapiol, a carvona e o limoneno, nas porcentagens de $14,4 \%, 55,2 \%$ e $16,6 \%$, respectivamente [27]. O d-limoneno é um terpenoide que apresenta forte ação repelente e de inibição da reprodução sobre lagartas-de-cartucho, pernilongos, besouros fitófagos e pragas de grãos armazenados, sendo utilizado comercialmente na formulação de diversos inseticidas agronômicos, não apresentando toxicidade aos animais homeotermos [28]. Esse composto fortemente aromático atua no sistema nervoso periférico, afetando as terminações sensoriais, o que causa forte desorientação nos insetos, levando-os à morte [29].

Em relação ao pó de A. graveolens, a dose estimada capaz de afetar a sobrevivência de $100 \%$ dos insetos foi de $0,25 \mathrm{~g}$ de pó de endro/10 g de grãos, sem mortalidade na testemunha (Figura 2).

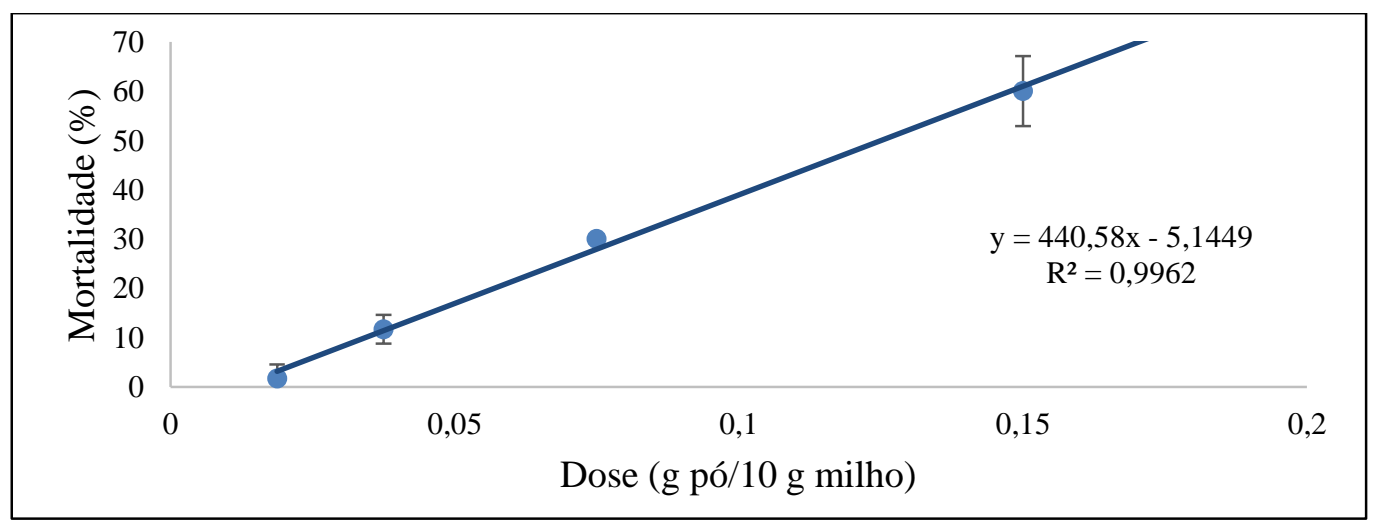

Figura 2: Mortalidade de Sitophilus zeamais tratado com o pó de Anethum graveolens. 
A DL ${ }_{50}$ obtida pelo método probit foi de $1,33 \mathrm{~g}$ de pó/ $10 \mathrm{~g}$ de milho. Procópio et al. (2003) [18] obtiveram como melhor resultado para a mortalidade de $100 \%$ dos insetos S. zeamais, 0,3g de pó/20 $\mathrm{g}$ de milho da espécie $C$. ambrosioides.

Os valores de $\mathrm{DL}_{50}$ encontrados para o extrato e para o pó A. graveolens evidenciam o maior potencial inseticida do pó em relação ao extrato. Este fato relaciona-se com algumas limitações dos extratos não aplicáveis aos pós, como a falta de controle de qualidade, baixa estabilidade dos compostos orgânicos presentes nas soluções e o não monitoramento de possíveis substâncias tóxicas presentes nas plantas ou resultantes da decomposição dos produtos durante sua manipulação [30] além da rápida degradação (por luz e/ou calor) e período curto de viabilidade [31].

$\mathrm{O}$ efeito do sinergismo das substâncias presentes em A. graveolens protegem as plantas, e sua ação inseticida também está ligada à inibição da enzima acetilcolinesterase nos insetos, prejudicando a atividade muscular e podendo levá-los a morte $[9,17,19,20]$. Monoterpenos, como o limoneno, podem agir diretamente no sistema nervoso dos insetos atingindo, além da acetilcolinesterase, canais de cloreto e sódio, receptores de octopamina e tiramina [15].

$\mathrm{O}$ extrato de $A$. indica apresentou atividade inseticida, porém, a dose necessária para atingir $50 \%$ de mortalidade $\left(\mathrm{DL}_{50}\right)$ dos insetos de $S$. zeamais foi mais alta $\left(325,4 \mu \mathrm{L} /\right.$ placa) em relação a $\mathrm{DL}_{50}$ de A. graveolens (121,5 $\pm 8,78 \mu \mathrm{L} /$ placa) (Figura 3).

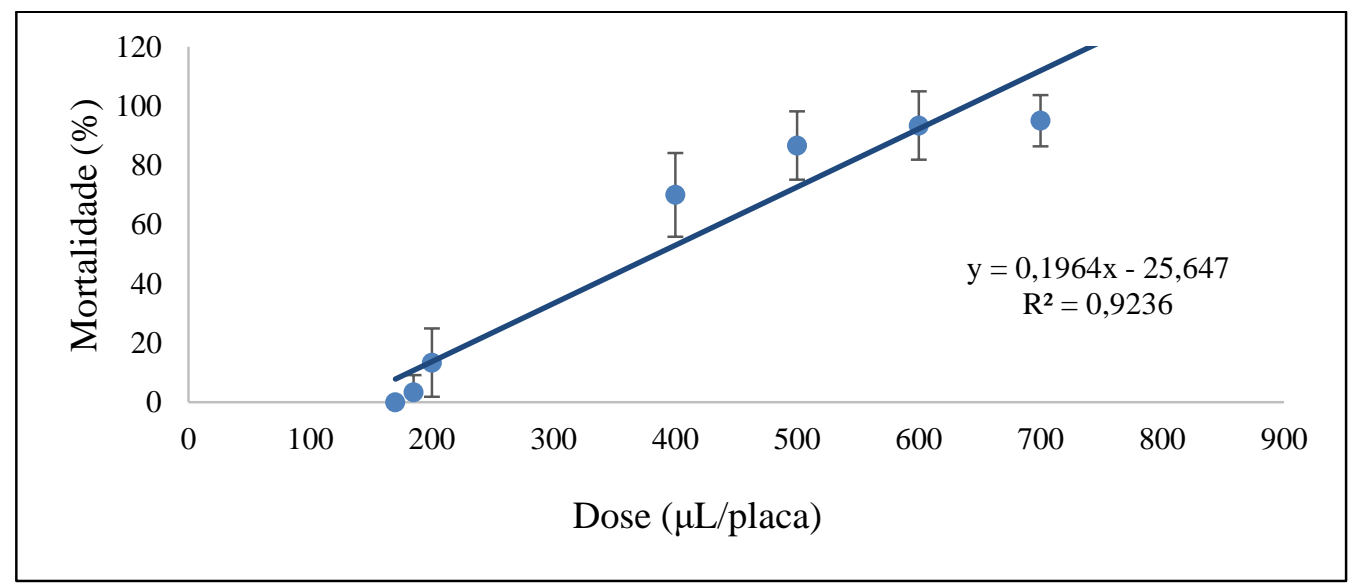

Figura 3: Mortalidade de Sitophilus zeamais com extrato de Azadirachta indica.

A família Meliaceae vem se destacando dentre as famílias botânicas tanto pelo número de espécies vegetais com atividade inseticida como pela eficiência de seus extratos, todas apresentam propriedades bioinseticidas inibindo a alimentação e bloqueando o crescimento de insetos, desde a etapa de quitinogênese ou de formação/regeneração do exoesqueleto durante a muda ou ecdise [32, 33]. Os mecanismos de ação da azadiractina também ocorrem por efeito antialimentar via oral, inibindo a atividade dos receptores de sensibilidade gustativa, modificando a ingestão normal de alimentos e a capacidade alimentar prospectiva dos insetos, o que permite a ingestão dos princípios ativos do nim juntamente com o alimento, conduzindo à inanição e à morte [34].

A azadiractina, limonóide encontrado na espécie $A$. indica, exerce influência no funcionamento de glândulas endócrinas controladoras da metamorfose de $S$. zeamais, sendo capaz de impedir o desenvolvimento larval já que, provoca alteração na atividade neuroendócrina de hormônios morfogenéticos, como a ecdisona. Além do mais, a redução do "turnover" do material neurosecretório, provocada pela azadiractina, diminui os níveis do hormônio, inibindo o desenvolvimento dos jovens e apresenta propriedade fagoinibidora $[10,14,19]$.

\subsection{Avaliação do potencial repelente}

Doses próximas a $1,35 \pm 0,5 \mu \mathrm{L} / \mathrm{cm}^{2}$ do extrato de Anethum graveolens foram suficientes para repelir 100\% dos insetos S. zeamais (Figura 4). 


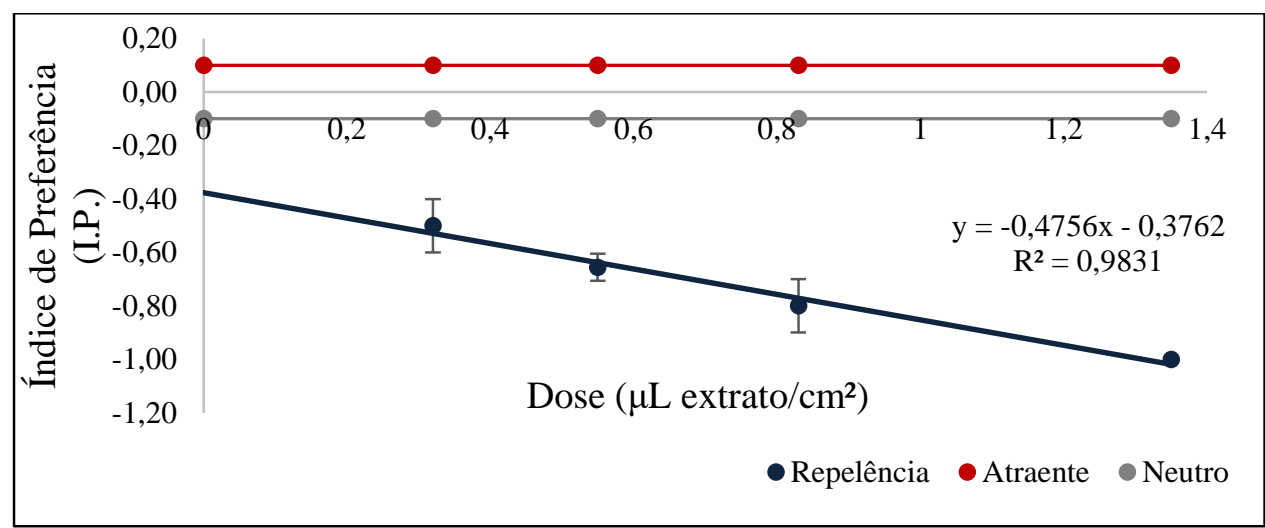

Figura 4: Efeito repelente do extrato de Anethum graveolens.

O efeito de repelência das plantas sobre insetos ocorre devido às substâncias químicas presentes em sua composição. Quando sofrem herbivoria, as plantas liberam substâncias com efeito repelente, como os alomônios que as protegem de ataques mais severos [35]. Por parte dos insetos, a repelência é uma reação do sistema sensorial ativada pela presença de quimiorreceptores, localizados na maioria das vezes em suas antenas, que detectam a presença de substâncias indesejáveis, provocando esse efeito em condições desfavoráveis [36]. De um modo geral, o endro possui em sua composição química substâncias do grande grupo de terpenos com ação repelente $[16,17]$.

A atividade repelente do pó de A. graveolens começou a partir da dose de 0,15 g (Figura 5).

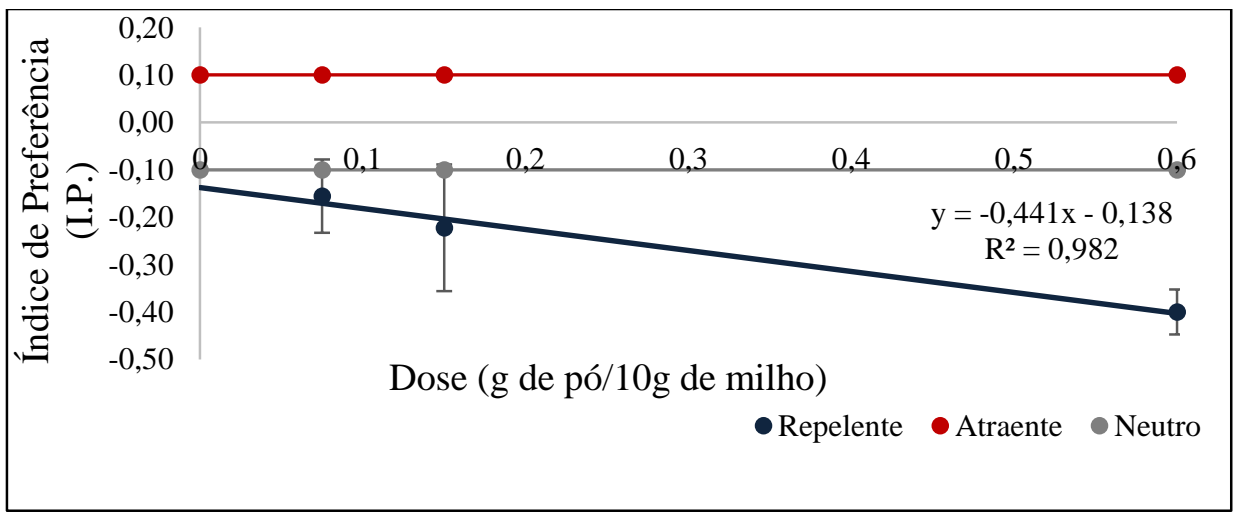

Figura 5: Efeito repelente do pó de Anethum graveolens.

A atividade repelente de $A$. indica foi nas doses de $2,29 \pm 0,5 \mu \mathrm{L} / \mathrm{cm}^{2}$ e $3,95 \pm 0,6 \mu \mathrm{L} \mu \mathrm{L} / \mathrm{cm}^{2}$. Porém as doses menores, $0,64 \pm 0,5 \mu \mathrm{L} / \mathrm{cm}^{2}$ e $1,37 \pm 0,5 \mu \mathrm{L} / \mathrm{cm}^{2}$, foram atraentes à $S$. zeamais (Figura 6).

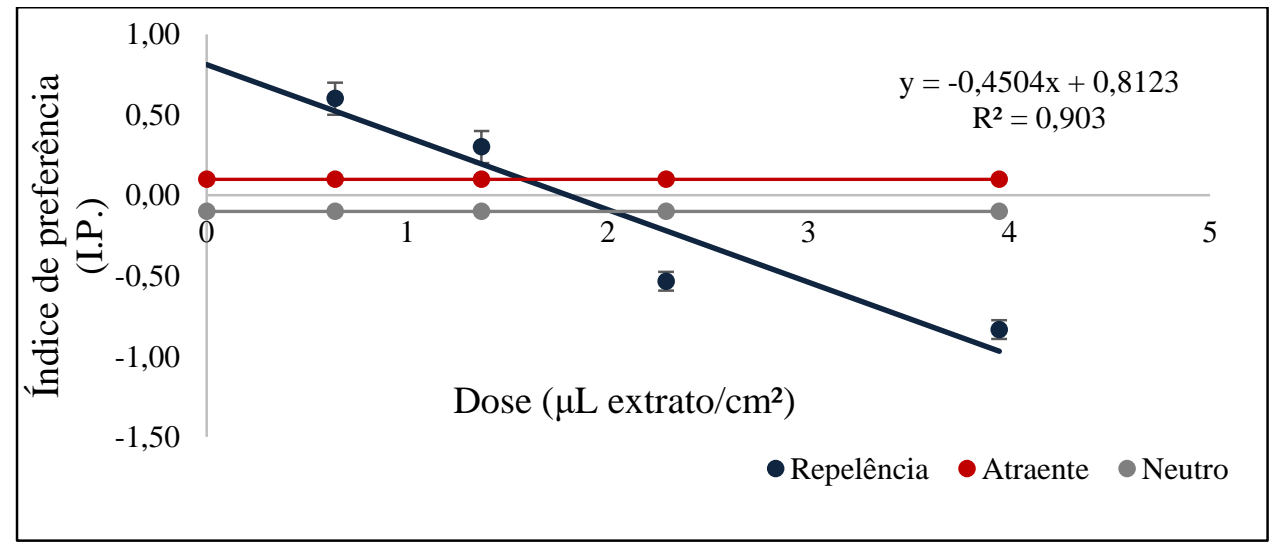

Figura 6: Efeito repelente do extrato de Azadirachta indica. 
Evidencia-se respostas diferentes em relação aos efeitos de atração e repelência de $S$. zeamais com A. indica conforme varia-se a dose de extrato. A literatura também aponta a existência de divergências entre os resultados para essa espécie. Lima-Mendonça et al. (2013) [21] constataram atividade neutra para o pó de A. indica na repelência de $S$. zeamais, enquanto Neto et al. (2019) [12] observaram a repelência de Callosobruchus maculatus em todas as doses decrescentes de pó de $A$. indica testadas.

Seu efeito repelente produz mudanças no comportamento, afetando a reprodução por inibição ou supressão da oviposição [37]. A azadiractina é muito instável em meios ácidos e alcalinos, em altas temperaturas e na presença da luz. Quando exposta a temperaturas acima de $50^{\circ}$ por 24 horas, sua ação inseticida pode ser reduzida em até $10 \%$ [38].

A azadiractina, componente majoritário de $A$. indica, apresenta diferentes efeitos sobre cerca de 30 espécies de insetos pertencentes a diversas ordens, dependendo muito da concentração, do método de extração do OE ou pó e até da forma de colheita da espécie [14, 22]. Além disso, essa substância pode afetar cerca de 200 espécies de insetos-praga, causando efeitos mínimos ou ausentes nos hospedeiros e predadores naturais dos insetos alvo, aumentando a segurança em sua utilização [19].

Tanto a ação de repelência quanto o efeito inseticida devem ser consideradas no controle de pragas de grãos armazenados, pois, quanto maior a repelência menor será a infestação, resultando na redução ou supressão da postura, bem como do número de insetos emergidos [39].

\section{CONCLUSÃO}

A. graveolens e A. indica possuem substâncias que afetam os gorgulhos e têm potencial para compor a formulação de inseticidas botânicos com a vantagem de serem eficientes, seguros e seletivos. Sugerem-se estudos mais detalhados a respeito da relação entre a composição química dos OEs com a atividade específica sob $S$. zeamais, bem como a influência de fatores externos no resultado, como método de extração dos OEs e condições laboratoriais para o inseto.

\section{AGRADECIMENTOS}

UNOESC - Universidade do Oeste de Santa Catarina.

\section{REFERÊNCIAS BIBLIOGRÁFICAS}

1. Artuzo FD, Foguesatto CR, Souza ÂRL, Silva LX. Costs management in maize and soybean production. Rev Bras Gestão Negócios. 2018 Apr-Jun;20(2):273-294, doi: 10.7819/rbgn.v20i2.3192

2. Sandhu H, Scialabba NE, Warner C, et al. Evaluating the holistic costs and benefits of corn production systems in Minnesota, US. Sci Rep. 2020;10:3922, doi: 10.1038/s41598-020-60826-5

3. Ojo JA, Omoloye AA. Development and Life History of Sitophilus zeamais (Coleoptera: Curculionidae) on Cereal Crops. Adv Agric. 2016;7836379, doi: 10.1155/2016/7836379

4. Muzemu S, Chitamba J, Goto S. Screening of stored maize (Zea mays L.) varieties grain for tolerance against maize weevil, Sitophilus zeamais (Motsch.). Int J Plant Res. 2013;3(3):17-22, doi: 10.5923/j.plant.20130303.01

5. Rosa JS, Oliveira L, Sousa RMOF, Escobar CB, Fernandes-Ferreira M. Bioactivity of some Apiaceae essential oils and their constituents against Sitophilus zeamais (Coleoptera: Curculionidae). Bull Entomol Res. 2019 Dec;1-11, doi: doi.org/10.1017/S0007485319000774

6. Santos VSV, Cunha JR, Silva PHS. Atividade ovicida e repelente de pó de citronela sobre o caruncho do feijão-caupi. Rev Verde Agroecol Desenv Sust. 2018 Abr;13(2):146-149, doi: 10.18378/rvads.v13i2.5157

7. Prates LHF, Faroni LRA, Heleno FF, Queiroz MELR, Sousa AH, Silva MVA. Eugenol diffusion coefficient and its potential to control Sitophilus zeamais in rice. Sci Rep. 2019 Aug;9:11161, doi: $10.1038 / \mathrm{s} 41598-019-47562-1$

8. Trecha CO. Potencial de chinchilho (Tagetes minuta, Asteraceae) no manejo agroecológico de Diabrotica speciosa (Germar, 1824) (Coleoptera: Chrysomelidae) em batata [tese]. Pelotas: Universidade Federal de Pelotas; 2018. 134 p. 
9. Simões CMO, Schenkel EP, Gosmann G, Mello JCP, Mentz LA, Petrovick PR. 6. ed. Porto Alegre/Florianópolis: Editora da Universidade UFRGS/Editora da UFSC; 2010.

10. Bhavaniramya S, Vishnupriya S, Al-Aboody MS, Rajendran V, Baskaran D. Role of essential oils in food safety: Antimicrobial and antioxidant applications. Grain Oil Sci Technol. 2019 Mar;2(2):49-55, doi: 10.1016/j.gaost.2019.03.001.

11. Mann CM, Cox SD, Markham JL. The outer membrane of Pseudomonas aeruginosa NCTC 6749 contributes to its tolerance to the essential oil of Melaleuca alternifolia (tea tree oil). Lett Appl Microbiol. 2000 Jan;30(4):294-297, doi: 10.1046/j.1472-765x.2000.00712.x

12. Neto EPS, Andrade ABA, Costa EM, Macarajá PG, Santos AB, Santos JLG, Pimenta TA. Effect of neem powder (Azadirachta indica A. Juss) on the control of cowpea weevils [Callosobruchus maculatus (F.) (Coleoptera: Bruchidae)] in cowpea beans. J Exp Agric Int. 2019 Jan;30(2):1-7, doi: 10.9734/JEAI/2019/46051

13. Schneider LCL, Silva CV, Conte H. Toxic effect of commercial formulations of neem oil, Azadirachta indica A. Juss., in pupae and adults of the sugarcane borer, Diatraea saccharalis F. (Lepidoptera: Crambidae). Arq Inst Biol. 2017 May;84:1-8, doi: 10.1590/1808-1657000432014

14. Liu ZL, Goh SH, Ho SH. Screening of Chinese medicinal herbs for bioactivity against Sitophilus zeamais Motschulsky and Tribolium castaneum (Herbst). J Stored Prod Res. 2007;43:290-296, doi: 10.1016/j.jspr.2006.06.010

15. Najafzadech R, Ghasemzadeh S, Mirfakhraie S. Effect of essential oils from Nepeta crispa, Anethum graveolens and Satureja hortensis against the stores-product insect "Ephestia kuehniella (Zeller)". J Med Plants By-products. 2019 Jun;2:163-169, doi: 10.22092/JMPB.2019.120494

16. Moraes RP, Correa IC, Vanin AB, Oliveira DL. Potencial tóxico dos óleos essenciais de endro (Anethum graveolens) e nim (Azadirachta indica A. Juss) frente Artemia salina. Braz J Develop. 2019 Apr;5(4):3834-3838.

17. Garcez JJ. Obtenção do extrato volátil de sementes de Anethum graveolens L. por diferentes técnicas extrativas [dissertação]. Porto Alegre: Pontifícia Universidade Católica do Rio Grande do Sul; 2016. $104 p$.

18. Procópio SO, Vendramim JD, Júnior JIR, Santos JB. Bioatividade de diversos pós de origem vegetal em relação a Sitophilus zeamais Mots. (Coleoptera: Curculionidae). Ci Agrotéc. 2003 Nov;27(6):1231-1236.

19. Júnior CV. Terpenos com atividade inseticida: uma alternativa para o controle químico de insetos. Quím Nova. 2003 Oct;26(3):390-400.

20. Fazolin M, Estrela JLV, Catani V, Alécio MR, Lima MS. Propriedade inseticida dos óleos essenciais de Piper hispidinervum C. DC.; Piper aduncum L. e Tanaecium nocturnum (Barb. Rodr.) Bur. \& K. Shum Sobre Tenebrio molitor L., 1758. Ci Agrotéc. 2007 Feb;31(1):113-120.

21. Lima-Mendonça A, Broglio SMF, Araújo AMN, Lopes DOP, Dias-Pini NS. Efeito de pós vegetais sobre Sitophilus zeamais (Mots., 1855) (Coleoptera: Curculionidae). Arq Inst Biol. 2013 Mar;80(1):91-97.

22. Silva AL, Chavez FCM, Lameira RC, Bizzo HR. Rendimento e composição do óleo essencial de Piper aduncum L. cultivado em Manaus, AM, em função da densidade de plantas e épocas de corte. Rev Bras PI Med. 2013 Jul;15(4):670-674.

23. Filho E, Antunes LEG, Tiecker A, Dionello RG, Spolti P. Controle de Gorgulho-do-milho submetido ao tratamento térmico. Rev Bras Milho Sorgo. 2011;10(3):196-204, doi: 10.18512/19806477/rbms.v10n3p196-204

24. Santos JC, Faroni LRD, Simões RO, Pimentel MAG, Sousa AH. Toxicity of pyrethroids and organophosphorus insecticides to Brazilian populations of Sitophilus zeamais (Coleoptera: Curculionidae). Biosci J. 2009 25(6):75-81.

25. Vedovatto F, Valério Júnior C, Astofoli V, Mielniczki PAA, Roman S, Paroul N, Cansian RL. Essential oil of Cinnamodendron dinisii Schwanke for the control of Sitophilus zeamais Motschulsky (Coleoptera: Curculionidae). Rev Bras Pl Med. 2015;17(4):1055-1060, doi: 10.1590/1983-084x/14_143

26. Rodrigues AC, Wiater G, Puton BMS, Mielniczki-Pereira AA, Paroul N, Cansian RL. Atividade repelente e inseticida do óleo essencial de Baccharis dracunculifolia D.C. sobre Sitophilus zeamais Mots, 1855. Perspectiva. 2019 Mar;43(161):123-130.

27. Singh G, Maurya S, De Lampasona M, Catalan C. Chemical constituents, antimicrobial investigations, and antioxidative potentials of Anethum graveolens L. essential oil and acetone extract: part 52. J Food Sci. 2005 Apr;70(4):208-2015.

28. Karr LL, Drewes CD, Coats JR. Toxic effects of d-limonene in the earthworm Eisenia fetida (Savigny). Pesticide Biochem Physiol. 1990 Oct;16(2):175-186.

29. Ware GW, Whitacre DM. Introducción a los insecticidas. In: The Pesticide Book. 6. ed. Ohio: Meister Pro Information Resources; 2004. 194 p. 
30. Silva MB, Rosa MB, Brasileiro BG, Almeida V, Silva CCA. Desenvolvimento de produtos à base de extratos de plantas para o controle de doenças de plantas. In: Controle alternativo de pragas e doenças. Viçosa: EPAMIG/CTZM; 2005. p. 221-246.

31. Potenza MR. Produtos naturais para o controle de pragas. In: X Reunião Itinerante de Fitossanidade do Instituto Biológico - Café; 2004 out 19; Mococa, SP. Mococa: Instituto Biológico: 2004. p. 89-100.

32. Roel AR, Vendramim JD, Frighetto R, Frighetto N. Atividade tóxica de extratos orgânicos de Trichilia pallida sobre Spodoptera frugiperda. An Soc Entomol Brasil. 2000 Dec;29(4):1-6, doi: 10.1590/s030180592000000400021

33. Itioca CS. Bioatividade comparativa entre Melia azedarach (cinamomo) e Azadirachta indica (nim): letalidade para larvas de Aedes aegypti. Ci Rural. 2004;34(4):979-981.

34. Saito ML. As plantas praguicidas, alternativa para o controle de pragas na agricultura. Inf. Embrapa Meio Ambiente 2004. p. 1-3.

35. Striquer LP, Bervian CIB, Faveiro S. Ação repelente de plantas medicinais e aromáticas sobre Sitophilus zeamais (Coleoptera: Curculionidae). Ensaios e Ciência. 2006 Abr;10(3):55-62.

36. Da Silva JF, Melo BA, Pessoa EB, Neto AF, Leite DT. Extratos vegetais para o controle do caruncho-dofeijão Zabrotes subfaciatus (Boheman 1833) (Coleoptera: Bruchidae). Rev Verde Agroecol Desenv Sust. 2013;8(3):1-5.

37. Akinneye JO, Ogungbite OC. Insecticidal activities of some medicinal plants against Sitophilus zeamais (Motschulsky) (Coleoptera: Curculionidae) on stored maize. Arch Phytopathol Plant Protect. 2013 Feb;46(10):1206-1213, doi: 10.1080/03235408.2013.763614

38. Schmutterer H. Properties and potencial of natural pesticides from the neem tree, Azadirachta indica. Ann Rev Entomol. 1990;35:271-297.

39. Guimarães SS, Potrich M, Silva ERL, Wolf J, Pegorini CS, Oliveira TM. Ação repelente, inseticida e fagoinibidora de extratos de pimenta dedo-de-moça sobre o gorgulho do milho. Arq Inst Biol. 2014;81(4):322-328.

40. Koul O, Walia S, Dhaliwal GS. Essential oils as green pesticides: potential and constraints. Biopesticides Int. 2008;4:63-84. 